\title{
Technikaffinität und Fortbildungsinteresse von Auszubildenden der Pflegefachberufe: eine Onlinebefragung
}

\author{
Christian Buhtz ${ }^{1}$ Denny Paulicke ${ }^{1,2} \cdot$ Sebastian Hofstetter ${ }^{1}$ Patrick Jahn ${ }^{3}$ \\ Eingegangen: 5. August 2019 / Angenommen: 8. Januar 2020 / Online publiziert: 30. Januar 2020 \\ (c) Der/die Autor(en) 2020
}

\section{Zusammenfassung}

Hintergrund Digitale und assistive Technologien nehmen eine prominente Rolle in der Diskussion um Lösungsansätze für die Herausforderungen der Gesundheitsversorgung ein. Für den Transfer in den Pflegealltag ist es erforderlich, Akzeptanz zu schaffen und Pflegefachpersonen in den Prozess der Digitalisierung des Gesundheitswesens zu integrieren. Allerdings wird im Rahmen der Pflegeausbildung bisher kaum Wissen für einen kompetenten Umgang mit Technologie vermittelt. Auch die zukünftige Ausbildungs- und Prüfungsordnung (Pflegeberufegesetz) unterlässt entsprechende Vorgaben.

Ziel Die Online-Umfrage soll klären, welches Maß an Aufgeschlossenheit und Fortbildungsinteresse bei Pflegeschülerinnen und Pflegeschülern zu digitalen und assistiven Technologien vorhanden ist.

Methode Eine Einladung zur Teilnahme wurde per E-Mail an 277 Pflegeschulen in den neuen Bundesländern verschickt. Der Fragebogen wurde von der Forschergruppe entwickelt und vorab getestet.

Ergebnisse Die Nutzung von Computern können sich 94,7\% der insgesamt 415 Befragten im Klinik- oder Praxisalltag vorstellen, die Nutzung von Tablets $61,9 \%$ und die Nutzung von Smartphones 51,1\%. Je zwei Drittel schätzen ihre Aufgeschlossenheit zu Technologie als hoch und ihr Wissen diesbezüglich als niedrig ein. Der Aussage, dass ihnen Schulungen zum bedarfsgerechten Einsatz technischer Lösungen fehlen, stimmen 71,8\% der Auszubildenden zu. Dies geht einher, mit einem hohen Fortbildungsinteresse der Auszubildenden, das je nach angebotenen Thema, von 64,8-83,4\% der Befragten geäußert wird.

Schlussfolgerung Der Bedarf und die Bereitschaft einer grundständigen Qualifizierung zu digitalen und assistiven Technologien im Rahmen der Pflegeausbildung - aber auch darüber hinaus - werden in dieser Befragung deutlich. Diese Kompetenzen müssen gezielt an einem praxisnahen Lernort („Future Care Lab“) vermittelt werden, nur so kann die digitale Transformation auf die Unterstützung qualifizierter professioneller Anwender aufbauen; andernfalls drohen langfristige Barrieren und Implementierungshindernisse.

Schlüsselwörter Ausbildung $\cdot$ Pflege $\cdot$ Digitalisierung $\cdot$ Assistive Technik $\cdot$ Robotik

Zusatzmaterial online Zusätzliche Informationen sind in der Onlineversion dieses Artikels (https://doi.org/10.1007/s16024020-00337-5) enthalten.

$\triangle$ Christian Buhtz

christian.buhtz@medizin.uni-halle.de

1 Dorothea Erxleben Lernzentrum Halle (DELH), Medizinische

Fakultät, Martin-Luther-Universität Halle-Wittenberg,

Magdeburger Straße 12, 06112 Halle (Saale), Deutschland

2 Internationale Graduiertenakademie, Institut für Gesundheits- und Pflegewissenschaft, Medizinische Fakultät, Martin-Luther-Universität Halle-Wittenberg, Halle (Saale),

Deutschland

3 Institut für Gesundheitswissenschaften, Abteilung Pflegewissenschaft, Medizinische Fakultät Tübingen, Tübingen, Deutschland 


\section{Affinity for technology and interest in advanced education of students in nursing professions: an online survey}

\section{Abstract}

Background Digital assistive technologies play a prominent role in the discussion about future solution approaches to the challenges of healthcare. For transfer into routine daily care it is necessary to achieve acceptance and to integrate the nursing personnel into the process of digitization of the healthcare system; however, hardly any skills in dealing with these technologies have been taught in the context of nursing education to date. The future training and examination regulations for the implementation of the Nursing Profession Act also fail to include corresponding provisions.

Aims This online survey was intended to clarify the extent of receptiveness and interest for advanced education in technical and digital assistive systems among nursing students.

Method An invitation to participate was sent by e-mail to 277 nursing schools in the new federal states of Germany. The questionnaire was developed and pretested by this research group.

Results For $94.7 \%$ of the 415 interviewees familiar systems, such as computers are conceivable in everyday working life, the use of tablets $61.9 \%$ and the use of smartphones $51.1 \%$. Of the participants two thirds rated their receptiveness to technology as high and knowledge of it as low. Of the trainees $71.8 \%$ agreed with the statement that they lack training in the needs-oriented use of technical solutions. This was accompanied by a high level of interest among trainees in continuing training, which was expressed by $64.8-83.4 \%$ of those surveyed depending on the topic on offer.

Conclusion The need and willingness for a basic qualification in digital and assistive technologies within the nursing education but also beyond was apparent from this survey. These competencies need to be successfully communicated through a practice-based approach (Future Care Lab), because the digital transformation needs to be built on the support of qualified professional users; otherwise, long-term barriers and obstacles to implementation pose a threat.

Keywords Nursing education $\cdot$ Nursing care $\cdot$ Digitization $\cdot$ Assistive technology $\cdot$ Robotics

\section{Einleitung}

Das deutsche Gesundheits- und Pflegesystem steht vor der Herausforderung, die zunehmende Anzahl pflegebedürftiger Menschen zu versorgen (Grünheid 2015; Rothgang und Müller 2018). Der dadurch steigende Bedarf an Pflegekräften verschärft sich weiter durch die Schwierigkeit, offene Stellen zu besetzen (Rothgang und Müller 2018). Zusätzliche Probleme sind die Überlastung der pflegenden Angehörigen und deren daraus resultierende eigene gesundheitliche Gefährdung (Rothgang und Müller 2018). Die Organisation for Economic Co-operation and Development (OECD) formuliert mit ,ageing in place“ das Ziel, ältere Menschen möglichst lange am Ort ihrer Wahl leben zu lassen, und auch im Ziel 3 „Ein gesundes Leben für alle Menschen jeden Alters gewährleisten und ihr Wohlergehen fördern“ der nachhaltigen Entwicklungsziele bis 2030 der WHO wird diese Zielstellung erneuert (Generalversammlung der Vereinten Nationen (UN) 2015; Mestheneos 2011). Diese Zielstellung muss im Kontext der genannten Schwierigkeiten und Herausforderungen betrachtet werden.

Mit dem Einsatz von digitalen und assistiven Technologien sind zur Lösung der oben genannten Probleme große Hoffnungen verbunden. Aus diesem Grund sind sie häufig Gegenstand der Forschung (Fachinger 2012; Haux 2016). Im häuslichen Bereich können hier Heimautomation bzw. SmartHome, aber auch Telepräsenzsysteme genannt wer- den. Ebenso sind robotische Systeme ein prominenter Bestandteil der angestrebten Lösungsszenarien (Bundesministerium für Bildung und Forschung [BMBF] 2018; Goeldner et al. 2015).

Die Formen der digitalen und assistiven Technologien sind vielfältig und durch die technische Entwicklung und Forschung einem ständigen Wandel unterworfen, weshalb eine einheitliche trennscharfe Unterscheidung und Kategorisierung der Systeme bisher noch fehlt (Meißner 2017). Selbst der Begriff der Assistenz im Kontext von Technologie ist nicht klar definiert bzw. wird unterschiedlich verstanden und lässt sich mangels eines Bezugsrahmens nicht eindeutig bestimmten Funktionalitäten zuordnen (Krings und Weinberger 2017). Der Transfer der Systeme von einzelnen Forschungsprojekten in die Regelversorgung ist ebenso noch nicht geglückt und bedarf einer breiteren und evidenzbasiert ausgerichteten Erforschung (Zegelin und Meyer 2018).

Die Gruppe der Nutzer ist heterogen; der Nutzerbegriff selbst bleibt unklar und wird in der Technikentwicklung meist nicht hinterfragt (Deisner et al. 2018). Nutzer lassen sich als Triade in einem Beziehungsdreieck aus Pflegeempfängern, Angehörigen und Pflegefachpersonen zeichnen. Sie sind dabei von einer grundlegenden Offenheit und Akzeptanz gegenüber der Nutzung digitaler und assistiver Technologien gekennzeichnet (Eggert et al. 2018). Diese Menschen sind als Nutzer von Technologie in ein komple- 
xes soziotechnisches System eingebettet, in welchem sich Technologie, Praktiken der Nutzung und die jeweilige Institution gegenseitig ,konfigurieren“ (Deisner et al. 2018).

In der Pflege zielt der Einsatz von Technologien u.a. darauf $a b$, den Pflegenden zeitliche und räumliche Unabhängigkeit zu bieten. Dies gilt gleichermaßen für die Pflegebedürftigen. Pflegepersonen vereinen vier Nutzerrollen in einer Personengruppe: Sie sind Anwender in dem Sinne, dass sie ein Gerät direkt bedienen, um eine pflegerische Maßnahme damit zu unterstützen, beispielsweise ein Mobilisierungsbett als Aufstehhilfe; als Administratoren warten und konfigurieren sie Geräte, welche von anderen Personen für pflegerische Belange genutzt werden, beispielsweise Telepräsenzsysteme für die Televisite; in dritter Instanz empfehlen sie auf Grundlage vorher erhobenen Unterstützungsbedarfs einem Pflegebedürftigen oder seinen Angehörigen ein Gerät zur Nutzung, beispielsweise einen automatischen Tablettenspender. Somit sind sie, in ihrer vierten Rolle, meist auch die ersten Ansprechpartner bei technischen Problemen und Fragen. Wenn bei den Pflegepersonen eine geringe Akzeptanz gegenüber der jeweiligen Technik vorliegt oder diese als nichtbedarfsgerecht angesehen wird, wird sie in diesem Sinne nicht genutzt und eingesetzt werden (Beer et al. 2011).

Die in der Literatur vorzufindenden theoretischen Modelle zur Akzeptanz von Technologien, wie die ,theory of reasoned action (TRA)“, das „technology acceptance model (TAM)“, die „unified theory of acceptance and use of technology (UTAUT)“ und deren Derivate und Erweiterungen unterscheiden zwischen der vom Anwender wahrgenommen Nützlichkeit (,perceived usefulness") und der Nutzbarkeit (,perceived ease of use") einer Technologie (Venkatesh et al. 2003; Davis et al. 1989). Die Nutzbarkeit meint u. a. eine für die anvisierte Nutzergruppe geeignete Bedienoberfläche eines Geräts. Untersuchungen zu den genannten Modellen zeigen, dass die Nutzbarkeit nur einen geringen Einfluss auf die Technikakzeptanz hat, also die Intention, ein Gerät oder eine Technologie zu nutzen (Davis et al. 1989). Andererseits hat die vom Anwender wahrgenommene Nützlichkeit den stärksten Einfluss auf die Akzeptanz von Technologie. Pflegende als Anwender müssen also von Sinn und Zweck einer Technologie überzeugt sein, weshalb eine strukturierte Hin- und Einführung zu den Systemen entscheidend für die Integration der Technologie in bestehende Prozesse der pflegerischen Versorgung ist.

Pflegefachpersonen müssen sich in einem zunehmend technologiegeprägten Umfeld zurechtfinden und dieses effektiv mitgestalten. Die gezielte Anwendung von digitalen und assistiven Technologien für die Verbesserung der Pflegequalität stellt dabei die Basis dar. Der Einfluss von Pflegefachpersonen bei der Konzeption und Auswahl der im Pflegealltag eingesetzten Technologien ist jedoch häufig gering, und pflegerisch-fachliche Interessen werden dabei zu selten fokussiert (Hergesell 2018). Ebenso geht ihre Beteiligung an der Entwicklung von Technik häufig nicht über die diskursive Ebene im Sinne von Anwender-Feedbacks hinaus (Deisner et al. 2018).

Für den Transfer von technischen Elementen in den Pflegealltag und die Nutzerakzeptanz ist es daher unabdingbar, dass die zukünftigen Nutzer aktiv in den Entwicklungs- und Integrationsprozess einbezogen werden und die pflegerischfachlichen Interessen sowie deren Ideen und Erwartungen berücksichtigt werden (Hergesell und Maibaum 2016; Deisner et al. 2018).

Der Zusammenhang zwischen Qualifizierung und Einstellung zur aktiven Nutzung von Technik wurde bisher nur in geringem Maße untersucht (Hielscher und Richter 2014). Einführende Qualifizierungen im Bereich assistive Technik für die Pflege sind vermutlich auch aus diesem Grund bisher nicht flächendeckend etabliert (Bearing Point 2017). In der Ausbildungs- und Prüfungsordnung der Gesundheitsund Krankenpflege sowie Altenpflege (PflAPrV) sind Lehreinheiten zu digitalen und assistiven Technologien bisher nicht vorhanden (Paulicke et al. 2018). In der Fassung von 2018, die im Rahmen der generalisierten Pflegeausbildung seit 2020 in Kraft ist, wird in der Anlage der Verordnung, die sich mit Kompetenzen für Prüfungen beschäftigt, die Digitalisierung ausschließlich im Kontext der Dokumentation und „Assistenzsysteme“ nur im Kontext der Wiedererlangung von Alltagskompetenzen erwähnt (PflAPrV). In welcher Form und zu welchen Rahmenbedingungen diese zu vermitteln sind, bleibt dort offen. Neben der Ausbildung mangelt es auch in der pflegerischen Fort- und Weiterbildung an Bildungsangeboten zu digitalen und assistiven Technologien (Roland Berger GmbH 2017).

Ammenwerth und Hackl definierten auf Grundlage einer Expertenbefragung 24 Kernkompetenzen der Pflegeinformatik (2019). Diese weisen jedoch kaum einen Bezug zu Anwendungsfällen in der pflegerischen Praxis auf, sondern konzentrieren sich verstärkt auf Themen der Informatik und IT-Anwendungen. Dies könnte darauf zurückzuführen sein, dass in der Befragung die Pflegenden, welche die Technik in der Patientenversorgung tatsächlich zur Anwendung bringen, nicht berücksichtigt wurden. Die Bereitstellung von Wissen zu bereits verfügbaren Produkten und Dienstleistungen ist eine wichtige Aufgabe. Zusätzlich besteht die Notwendigkeit, Kompetenzen zur bedarfsgerechten und nutzbringenden Anwendung assistiver Technologien aufzubauen, die über das Erlernen von Fertigkeiten und Fähigkeiten zur Handhabung hinausgehen.

Zentrale Ansatzpunkte sind dabei die Verknüpfung von Wissenserwerb und der sich daraus ergebende Praxistransfer. Die Einbindung von digitalen und assistiven Technologien in die Ausbildung von Schülerinnen und Schülern der Pflegeberufe dient dabei als Basis, um die Auszubildenden als „early adopter“ und „gatekeeper“ für die Zukunft der 
Versorgung gewinnen zu können. Dabei ist nicht der Einsatz von IT-Technik und digitalen Lernmitteln (z. B. Laptops oder smarte Whiteboards) als Lehrmethode bzw. Mittel zur Wissensvermittlung gemeint. Neben dem Wissen über bereits vorhandene Systeme ist der Aufbau entsprechender Fertigkeiten und weiterer Kompetenzen zu kritischer und reflektierter Aneignung von Technik und zum ethischen und sozialverträglichen Einsatz sowie der Integration in den Versorgungsprozess elementar. Ebenso sind Kompetenzen erforderlich, um an der Weiterentwicklung dieser Technologien zu partizipieren.

Im Diskurs fehlen bisher eine klare Trennung zwischen Informatik und Pflegepraxis sowie eine konkrete Beschreibung und Ausdifferenzierung der Rollen und Kompetenzen dieser beiden Bereiche im Rahmen der Digitalisierung der Gesundheitsversorgung. Die Bestrebungen, ein neues bzw. zusätzliches pflegerisches Berufsbild, häufig als Pflegeinformatik bezeichnet, im Gesundheitswesen zu etablieren, erscheint nicht zielführend. Dies würde durch die verschärfte Arbeitsteilung zu parallelen Prozessen der Spezialisierung und Dequalifizierung unter den Pflegeberufen führen (Hielscher et al. 2015). Somit würden den bereits etablierten Pflegeberufen und insbesondere den akademisierten Pflegenden weitere Kompetenzen vorenthalten.

Gleichwohl sind Informatikerinnen und Informatiker notwendig, die Kenntnisse über die Prozesse in der Gesundheitsversorgung besitzen. Denkbar wäre es, ein Fachgebiet „Informatik für Gesundheitsversorgung“" zu etablieren und gleichzeitig mit der „Medizininformatik“ zu fusionieren, da hier vielfältige inhaltliche und kaum trennbare Überschneidungen vorhanden sind.

Da Technologie jeden Bereich der Pflege durchdringt, besteht die Notwendigkeit, allen Pflegenden Technologiekompetenz zu vermitteln und diese in bestehende Aus-, Fort- und Weiterbildungen zu integrieren (Hübner et al. 2017; Locsin 2017). Die Vermittlung sollte dem jeweiligen Qualifizierungsniveau und Einsatzfeld angepasst sein und trägt den Schwerpunkt der Anpassungen des Pflegeprozesses für eine effektive Integration der assistiven Technik. Die Einstellungen und Einschätzungen besonders von angehenden Pflegepersonen sind besonders wichtig, um die künftige Gestaltung des Bildungswesens auch mit diesem Themenfeld anzureichern.

Nach dem bisherigen Wissenstand und den Erfahrungen der Autoren gibt es keine Untersuchungen zu Bildungsangeboten für die Pflegeberufe oder deren Auszubildende im Kontext des praktischen Einsatzes von digitalen und assistiven Technologien.

\section{Zielsetzung und Fragestellung}

Um die Konzeption entsprechender Bildungsangebote für Auszubildende in den Pflegeberufen zu unterstützen, sollen das Wissen und die Einstellung dieser Zielgruppe zu digitalen und assistiven Technologien und damit verbundene Bildungsangebote erforscht werden. Hierfür wurde eine Onlinebefragung durchgeführt, um die Aufgeschlossenheit, das Wissen sowie das Fortbildungsinteresse hinsichtlich digitaler und assistiver Technologien bei den Auszubildenden in den Pflegeberufen zu ermitteln.

\section{Material und Methode}

Die Onlinebefragung ist dem Mixed-Method-Design angelehnt, welches sich aus drei Teilschritten zusammensetzt (Kuckartz 2014). Um den Fragebogen für die Auszubildenden entwickeln zu können, fanden in einem ersten Schritt Fokusgruppengespräche mit Vertreterinnen und Vertretern der Pflegefachberufe, der Therapieberufe und der Ärzteschaft statt. Begleitend dazu wurde eine Literaturrecherche über Bildungskonzepte zu assistiven Technologien im Alter durchgeführt (Paulicke et al. 2018; 2019b). Die Ergebnisse der Recherche und Fokusgruppen ermöglichten im nächsten Schritt die Konzeption eines Fragebogens. Dieser wurde von einer interdisziplinären Arbeitsgruppe, die sich aus den Fachbereichen Pflegewissenschaften, Informatik und Medizin zusammensetzt, erstellt. Im letzten und dritten Schritt erfolgte ein kognitiver Pretest mit Studierenden der Studiengänge „Evidenzbasierte Pflege“ und „Gesundheits- und Pflegewissenschaften“ der Martin-Luther-Universität Halle-Wittenberg, um sich der eigentlichen Zielgruppe, den Auszubildenden der Pflegeberufe, anzunähern. Im Ergebnis des Pretests wurde der Fragebogen unter dem Aspekt der Verständlichkeit hinsichtlich einiger Formulierungen angepasst.

Der Fragebogen wurde in deutscher Sprache verfasst und bestand aus Multiple- und Single-Choice-Fragen, Bewertungsfragen mit einer Skalierung nach Likert sowie offenen Fragen (Zusatzmaterial online: 1). Neben der Erhebung von soziodemografischen Daten wurden das Wissen und die Aufgeschlossenheit bezüglich der Anwendung von Technologien und die Fortbildungsbereitschaft zu technischen Lösungen erfragt. Die Auswahl der FragebogenItems mit Bezug zu spezifischen Technologien basiert auf früheren Untersuchungen (Klein et al. 2018; Buhtz et al. 2018). Die sechs Bereiche des Pflegebedürftigkeitsbegriffs ( 14 SGB XI) wurden in Frage 8 des Fragebogens (Zusatzmaterial online: 1) verwendet, um spezifische Technologien verständlich zu erfragen und hinsichtlich der Anwendungsbereiche zu kategorisieren: automatischer Medikamentenspender (Bereich: therapiebedingte Anforderungen), ein 
Mobilisierungsbett (Bereich: Mobilität), ein Bildtelefon (Bereich: Kommunikation), ein Hausnotrufsystem (Bereich: Sicherheit), ein höhenverstellbares Waschbecken (Bereich: Selbstversorgung) sowie ein Tageskalender mit Erinnerungsfunktion (Bereich: Gestaltung des Alltagslebens).

Die im Fragebogen verwendeten Begriffe für Technologien wurden ohne weitere Definitionen oder Erklärungen vorgegeben. Weiterhin wurde in Form je eines Bildes ein Kommunikationsroboter (als Pepper von Softbank Aldebaran Robotics, Paris, Frankreich) und ein Telepräsenzsystem (als Double von Double Robotics, Burlingame, CA, USA) dargestellt.

Der Feldzugang erfolgte über Alten- und Krankenpflegeschulen in Mecklenburg-Vorpommern, Brandenburg, Sachsen-Anhalt, Sachsen, Thüringen und Berlin. Diese regionale Eingrenzung ist durch die spezifische demografische Entwicklung in diesen Bundesländern begründet (Kreuter und Klingholz 2012). Basierend auf Zahlen zur Entwicklung der Bevölkerung, des Durchschnittsalters und des Zuwachses an Pflegebedürftigen befinden sich diese Bundesländer bereits in einer fortgeschrittenen Phase des demografischen Wandels, während die restlichen Bundesländer die demografische Spitze erst Jahre später erreichen werden (Grünheid 2015; Rothgang und Müller 2018).

Für die Recherche nach E-Mail-Adressen von Pflegeschulen wurden sowohl eine allgemeine Internetsuchmaschine als auch die Suchmasken der Internetseiten des Bundesministeriums für Familie, Senioren, Frauen und Jugend und pflege-kurse.de genutzt. Hierbei konnte eine vereinheitlichte Liste von Pflegeschulen generiert werden. Dazu zählen Altenpflegeschulen sowie Schulen der Gesundheits- und Krankenpflege. Ausgeschlossen wurden Schulen für Altenpflegeassistenz sowie Heilpraktikerschulen und heilpädagogische Bildungseinrichtungen. Daran anschließend wurden in zwei Wellen im Oktober und im Dezember 2017 an 277 auf diese Weise identifizierte Fachschulen für Pflegeberufe elektronische Informationsschreiben verschickt, die einen Link zu einer Onlinebefragung enthielten. Hierbei wurden - soweit möglich - die Schulleiterinnen und Schulleiter der jeweiligen Schulen mit der Bitte um Verteilung und Weiterleitung angeschrieben. Auf weitere Schritte der internen Verteilung wurde kein Einfluss genommen. Auch ist nicht bekannt, wie viele Schülerinnen und Schüler in den Einrichtungen eingeschrieben waren. Daher können keine Aussagen zur Anzahl der tatsächlich erreichten Auszubildenden und entsprechend auch nicht zur Rücklaufquote getroffen werden

Die Daten wurden mit der Onlinebefragungsplattform SoSci Survey erfasst und im Anschluss mit SPSS und R deskriptiv ausgewertet (SoSci Survey 2018; IBM 2017; $\mathrm{R}$ Core Team 2017). Die Berichterstattung richtet sich entsprechend der gewählten Methodik nach den Berichtselementen des STROBE Statement (Elm et al. 2007).

\section{Ergebnisse}

Der Onlinefragebogen wurde im Untersuchungszeitraum 580-mal aufgerufen. Es wurden 415 Fragebögen vollständig ausgefüllt, die Eingang in die Auswertung fanden. Aufgrund der Art des gewählten Feldzugangs gibt es keine Daten, auf deren Grundlage eine Rücklaufquote berechnet werden kann.

Der Großteil der Stichprobe ist mit 316 von 415 Personen weiblichen Geschlechts (Tab. 1). Die bis 20-Jährigen stellen mit fast der Hälfte den größten Teil der Befragten. Mehrheitlich $(66,3 \%)$ antworteten Schülerinnen und Schüler in der Altenpflegeausbildung. Weitere Ausbildungsrichtungen sind die Gesundheits- und Krankenpflege (30,4\%), Altenpflegehilfe (1,4\%), Gesundheits- und Kinderkrankenpflege $(0,5 \%)$ und andere Ausbildungen (1,2\%). Der mit Abstand größte Rücklauf kommt mit mehr als der Hälfte von Auszubildenden aus Sachsen-Anhalt. Beinahe alle $(97,1 \%)$ Befragten geben an, bereits praktische Erfahrungen im Umgang mit Patienten zu haben.

In der Erhebung wird erfragt, ob niederschwellige technische Lösungen im Bereich der häuslichen Pflege bekannt sind und inwieweit bereits Erfahrungen damit gemacht wurden (Abb. 1). Am wenigstens bekannt sind der Medikamentenspender mit Erinnerungsfunktion (15,9\%), das Bildtelefon $(29,2 \%)$, das höhenverstellbare Waschbecken $(29,9 \%)$

Tab. 1 Beschreibung der Stichprobe

\begin{tabular}{lr}
\hline & $\%$ \\
\hline Geschlecht $(n=415)$ & 76,1 \\
Weiblich & 23,9 \\
Männlich & 0,0 \\
Keine Angaben & \\
Alter $(n=415)$ & 49,4 \\
Bis 20 Jahre & 30,1 \\
21 bis 30 Jahre & 10,6 \\
31 bis 40 Jahre & 5,3 \\
41 bis 50 Jahre & 0,5 \\
Über 50 Jahre & 4,1 \\
Keine Angaben & \\
Bundesland $(n=415)$ & 9,6 \\
Sachsen & 53,0 \\
Sachsen-Anhalt & 12,3 \\
Berlin & 4,8 \\
Brandenburg & 1,9 \\
Mecklenburg-Vorpommern & 18,1 \\
Thüringen & 0,2 \\
Keine Angaben &
\end{tabular}




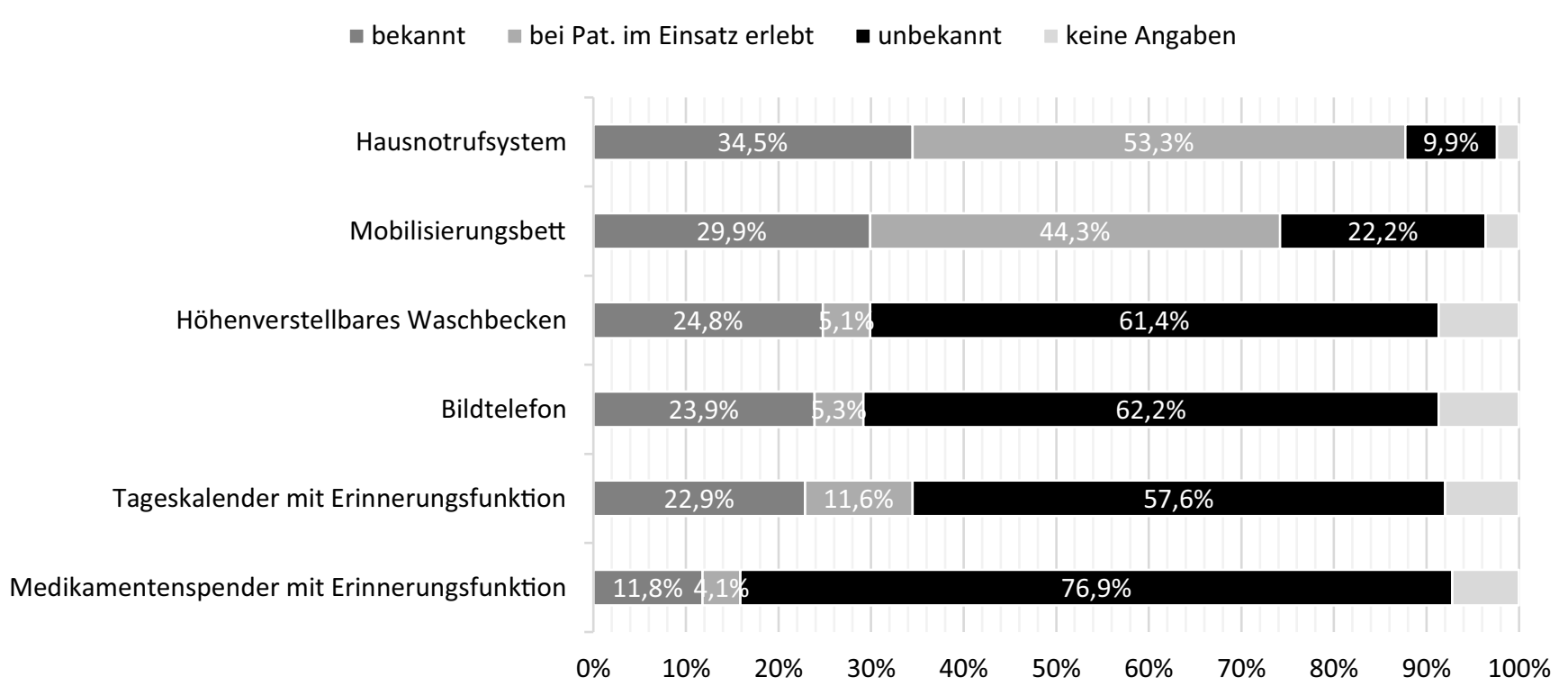

Abb. 1 Bekanntheit und Erfahrung mit technischen Lösungen $(n=415)$

und der Tageskalender mit Erinnerungsfunktion (34,5\%). Das Hausnotrufsystem ist den meisten Schülerinnen und Schülern bekannt $(87,8 \%)$ bzw. wurde bereits bei Patienten im Einsatz erlebt (53,3\%).

Die Nutzung von Computern können sich 94,7\% der Befragten im Klinik- oder im Praxisalltag vorstellen (Tab. 2). Als niederschwellige und bzw. bekanntere technische Systeme können sich $61,9 \%$ der Auszubildenden Tablets und $51,1 \%$ Smartphones als Unterstützung im Pflegealltag vorstellen. Dementgegen kann sich nur ein kleiner Teil der Befragten die Anwendung von komplexeren assistiven Technologien wie Datenbrillen (8,9\%), Virtual-Reality-Technologie $(7,7 \%)$ oder Spielekonsolen $(8,0 \%)$ vorstellen. Ein Videokonferenzsystem wäre für 22,9\% der Auszubildenden im Berufsalltag vorstellbar.

In den Settings Krankenhaus und stationäre Pflege sind komplexere und höher entwickelte assistive Technologien, wie ferngesteuerte Telepräsenzsysteme und autonome Ro-

Tab. 2 Vorstellbarkeit spezifischer Systeme im Klinik- oder im Praxisalltag

\begin{tabular}{lc}
\hline & $\%^{\mathrm{a}}$ \\
\hline Computer & 94,7 \\
Videokonferenzsystem & 22,9 \\
Smartphone & 51,1 \\
Tablet & 61,9 \\
Smartwatch & 19,3 \\
Fitness-Armband & 24,8 \\
Datenbrille & 8,9 \\
Digitalisierungsstift & 19,3 \\
Spielekonsole & 8,0 \\
Virtual-Reality-Technologie & 7,7 \\
\hline
\end{tabular}

${ }^{a} n=415$ boter, für etwa ein Drittel der Schülerinnen und Schüler vorstellbar, wobei der Roboter in der Vorstellbarkeit überwiegt (Tab. 3). Insgesamt sind beide Gerätearten in den insgesamt fünf abgefragten Settings der Gesundheitsversorgung für nur einen geringen Teil der Auszubildenden vorstellbar (23,9-46,5\%). Der Einsatz zur Sicherung der Selbstständigkeit im häuslichen Umfeld ist mit $46,5 \%$ für die Telepräsenz bzw. 42,2\% für einen Roboter noch das realistischste Szenario für die befragten Auszubildenden.

Ihre Aufgeschlossenheit zur Anwendung von computergestützter Technologie im Allgemeinen schätzen 67,7\% als hoch ein. Ihr Wissen hingegen bezüglich technischer Lösungen in Klinik- und Pflegealltag betrachten die Befragten mehrheitlich mit $66 \%$ als niedrig.

Von den befragten Schülerinnen und Schülern geben $79 \%$ an, keine Schwierigkeiten dabei zu haben, die Probleme und Bedarfe ihrer Patienten bezüglich der Integration von technischen Lösungen im häuslichen Umfeld zu erkennen. Die Aussagen, dass der Nutzen von technischen Lösungen ihnen unbekannt sei, wird von $34 \%$ bejaht und von $61,2 \%$ verneint. Das Wissen über Finanzierungsmöglichkeiten fehlt 69,2\% der Auszubildenden. Das Fehlen von Schulungen zum bedarfsgerechten Einsatz technischer Lösungen bemängeln 71,8\% der Auszubildenden. Ansprechpartner und Support werden von $69,4 \%$ vermisst.

In allen durch den Fragebogen vorgegebenen Bereichen geben mehr als die Hälfte der Befragten $(64,8-83,4 \%)$ ein hohes Fortbildungsinteresse an (Tab. 4). Besonders hohe Bereitschaft der Befragten besteht in den Bereichen der klinischen/pflegerischen Arbeitsorganisation (78,3\%), der klinischen/pflegerischen Therapie $(83,4 \%)$ und der Kommunikation mit Ärzten $(77,8 \%)$. 
Tab. 3 Vorstellbarkeit von Telepräsenz oder Roboter im Versorgungskontext

\begin{tabular}{|c|c|c|c|c|c|c|}
\hline & \multicolumn{3}{|c|}{ Telepräsenz $^{\mathrm{a}}$} & \multicolumn{3}{|c|}{ Roboter $^{\mathrm{b}}$} \\
\hline & \multicolumn{3}{|c|}{$\begin{array}{l}\text { Vorstellbar }(\%) \\
n=415\end{array}$} & \multicolumn{3}{|c|}{$\begin{array}{l}\text { Vorstellbar }(\%) \\
n=415\end{array}$} \\
\hline & $\mathrm{Ja}$ & Nein & k. A. ${ }^{\mathrm{c}}$ & $\mathrm{Ja}$ & Nein & k. A. ${ }^{c}$ \\
\hline Einsatz im Krankenhaus & 28,0 & 71,6 & 0,5 & 34,9 & 64,3 & 0,7 \\
\hline Einsatz in der stationären Pflege & 26,5 & 72,5 & 1,0 & 34,2 & 64,6 & 1,2 \\
\hline Einsatz in der ambulanten Pflege & 26,3 & 72,3 & 1,4 & 23,9 & 75,2 & 1,0 \\
\hline $\begin{array}{l}\text { Einsatz bei der hausärztlichen Be- } \\
\text { treuung in der Häuslichkeit }\end{array}$ & 31,6 & 66,7 & 1,7 & 31,1 & 67,7 & 1,2 \\
\hline $\begin{array}{l}\text { Einsatz zur Sicherung der Selbst- } \\
\text { ständigkeit im häuslichen Umfeld }\end{array}$ & 46,5 & 51,8 & 1,7 & 42,2 & 56,9 & 1,0 \\
\hline
\end{tabular}

Tab. 4 Interesse an Fortbildungen bezüglich technischer Lösungen

\begin{tabular}{|c|c|c|c|}
\hline $\begin{array}{l}\text { Fortbildungen und Informationsveranstaltungen bezüglich techn. Lösungen zur Unterstüt- } \\
\text { zung von } \ldots(n=415)\end{array}$ & Nein $(\%)$ & $\mathrm{Ja}(\%)$ & k. A. ${ }^{a}(\%)$ \\
\hline Klinische oder pflegerische Arbeitsorganisation & 19,8 & 78,3 & 1,9 \\
\hline Klinische oder pflegerische Therapie & 15,7 & 83,4 & 1,0 \\
\hline Klinische oder pflegerische Diagnostik & 20,5 & 76,9 & 2,7 \\
\hline Techn. Lösungen zur häuslichen Unterstützung von Patienten & 28,2 & 70,4 & 1,4 \\
\hline Techn. Lösungen zur häuslichen Unterstützung von pflegenden Angehörigen & 32,5 & 64,8 & 2,7 \\
\hline Kommunikation mit ärztlichen Kollegen & 20,2 & 77,8 & 1,9 \\
\hline Interprofessionelle Vernetzung und Kommunikation & 28,0 & 69,9 & 2,2 \\
\hline Vernetzung und Kommunikation mit Patienten und pflegenden Angehörigen & 24,6 & 73,0 & 2,4 \\
\hline
\end{tabular}

${ }^{a}$ Keine Angabe

\section{Diskussion}

Auszubildende der Alten- und Krankenpflege in den neuen Bundesländern zeigen eine große Aufgeschlossenheit gegenüber digitalen und assistiven Technologien. Das eigene Wissen darüber wird jedoch mehrheitlich als niedrig eingeschätzt wird. Es wurde deutlich, dass eine hohe Bereitschaft zur Teilnahme an Bildungsangeboten zum Themenbereich der digitalen und assistiven Technologien vorhanden ist und auch der Bedarf dazu gesehen wird.

Kritisch ist zu sehen, dass survey-typisch wenig über die Response-Rate der Befragten gesagt werden kann. Insgesamt muss diese aber als gering eingestuft werden, da 277 Schulen den Einladungslink mit dem Onlinefragebogen erhalten hatten. Trotz der großen Anzahl hat nur ein geringer Anteil den Fragebogen beantwortet. Dies könnte zum einen daran liegen, dass es keinen persönlichen Kontakt gab, oder dass zu wenig Interesse seitens der Schulleitungen bestand, den gesendeten Link an die Auszubildenden weiterzuleiten. Des Weiteren gaben auch einige Schulen an, keine MailAdressen von ihren Schülerinnen und Schülern zu kennen. Über das Antwortverhalten der einzelnen Schulen und Bundesländer kann keine Aussage getroffen werden, da hierzu keine Daten vorliegen. Weitere Vermutungen bezüglich des zurückhaltenden Antwortverhaltens sind der relativ große
Umfang des Onlinefragebogens und die Komplexität der darin enthaltenen Fragen.

Limitierend kann außerdem angemerkt werden, dass einige Fragen möglicherweise schwer verständlich waren. Ursächlich für einige Verständnisprobleme könnte dabei gewesen sein, dass den Befragten die einzelnen Technikbegriffe ohne vertiefende Definitionen oder weiterführende Erklärungen präsentiert wurden. Die Auswahl an im Fragebogen genutzten Technologiebegriffen ist begrenzt und deckt daher nur einen Teil des möglichen Spektrums an digitalen und assistiven Technologien ab. Ebenso resultiert diese Begrenzung daraus, dass im aktuellen Diskurs und Forschungsstand noch keine trennscharfe Unterscheidung zwischen den Geräten und Technologieformen getroffen werden kann und dass ebenso noch keine Klarheit darüber besteht, welche für den pflegerischen Bereich relevant und nützlich sind.

Die Ergebnisse zur Einordnung und Einschätzung der assistiven Technologien weisen Ähnlichkeiten zu Erhebungen für andere Zielgruppen in der Gesundheitsversorgung auf. Pflegende Angehörige von Menschen mit Demenz äußern ebenfalls einen Bedarf an Bildungsangeboten hinsichtlich digitaler und assistiver Technologien, wobei solche Angebote für diese hochrelevante Zielgruppe bisher nicht bestehen (Paulicke et al. 2019a, 2019b). Bezüglich der Aufge- 
schlossenheit zum Wissen über Technologien konnten keine vergleichenden Zahlen zu verwandten Stichproben gefunden werden. Innerhalb der eigenen Forschungsgruppe existiert jedoch eine ähnliche Befragung von Hausärzten (Buhtz et al. 2019): In dieser fiel die Selbsteinschätzung bezüglich Wissen (71\%, ,niedrig“) und Aufgeschlossenheit (53\%,,hoch“) ähnlich aus.

Auffallend war, dass der Großteil der Befragten hinsichtlich der Integration von technischen Lösungen im häuslichen Umfeld keine Schwierigkeiten hat, die Probleme und Bedarfe der Patientinnen und Patienten zu erkennen, sich aber gleichzeitig mit assistiven Techniken nicht genügend auskennt. Auch Geräte wie Medikamentenspender, die die Medikamente automatisch zu einer voreingestellten Zeit bedarfsgerecht ausgeben, sind den meisten Schülerinnen und Schülern unbekannt. Die Vorstellungskraft für den nutzbringenden Einsatz komplexerer technologiebasierter Systeme wie Robotik oder Telepräsenz ist eher gering. Es ist anzunehmen, dass dies v. a. darin begründet ist, dass das Thema assistiver Technologien im Lehrplan nicht verankert ist (Bearing Point 2017). Das Selbstvertrauen der Befragten in ihre Fähigkeit zur Bedarfseinschätzung und die damit verbundene Integration der Technologien müssen hinterfragt werden, da die assistiven Systeme zumeist unbekannt sind und deren Komplexität nicht adäquat eingeschätzt werden kann. Gleichwohl ist dieses Selbstvertrauen eine wichtige Ressource für Qualifizierung und Implementierung, da mit zunehmenden Wissen die Fähigkeit entsteht, Wissenslücken $\mathrm{zu}$ erkennen und die eigenen Kompetenzen realistischer zu reflektieren.

Die untersuchte Gelegenheitsstichprobe ist ausreichend groß und hinsichtlich der Geschlechterverteilung eine gute Annährung an die Gesamtpopulation. Obwohl Aussagen abgeleitet werden können, lässt sich eine Verzerrung der Stichprobe nicht grundsätzlich ausschließen. Somit kann die Stichprobe nicht uneingeschränkt als repräsentativ betrachtet werden. Da die Stichprobe zu einem großen Teil aus Auszubildenden der Altenpflege besteht, deren Tätigkeitsfelder im Vergleich zur Klinik mutmaßlich weniger technologiegeprägt sind, könnte das Ergebnis diesbezüglich verzerrt sein. Ebenso steht die Möglichkeit im Raum, dass insbesondere technikinteressierte Auszubildende sich von der Befragung angesprochen gefühlt haben.

\section{Schlussfolgerung}

In Anbetracht der hohen Erwartungshaltungen, die mit der Digitalisierung und dem Einsatz assistiver Technologien in der Gesundheitsversorgung einhergehen, deuten die Ergebnisse darauf hin, dass hinsichtlich der Ausbildung der größten Berufsgruppe im Gesundheitswesen erheblicher Nach- holbedarf besteht (Bearing Point 2017; Bundesministerium für Bildung und Forschung (BMBF) 2018).

Trotz der geringen Vorstellungskraft ist eine deutliche Bereitschaft zur Fort- und Weiterbildung unter den Befragten zu erkennen. Dieser Umstand und die Aufgeschlossenheit gegenüber digitalen und assistiven Technologien sind grundlegende Elemente zur effizienten Förderung eines Praxistransfers. Da für viele Pflegeszenarien bereits technische Hilfsmittel zur Verfügung stehen, müssen Schulungsprogramme und Bildungsangebote verstärkt in die Ausbildung integriert werden. Durch den ausbildungsintegrierten Einsatz werden Technologien transparent dargestellt, erfahrbar gemacht und der Praxistransfer erleichtert. Pflegefachpersonen nehmen dabei verschiedene Rollen ein, auf die sie vorbereitet werden müssen. Die Akzeptanz für den technischen Einsatz ist im Bereich der Entlastung von Routinetätigkeiten am höchsten (Roland Berger GmbH 2017). Die angesprochenen Kompetenzen müssen in die bestehenden Berufsbilder und -rollen integriert und neben der grundständigen Ausbildung und dem Studium, auch in Fort- und Weiterbildungen entsprechend dem jeweiligen Qualifizierungsniveau und Tätigkeitsfeld vermittelt werden (Hübner et al. 2017). Ziel sollte es sein, das Empowerment der Pflegenden zum adäquaten Einsatz und der Einschätzung von assistiver Technologie zu stärken. Neben der Vermittlung von Technikkompetenz bei allen in der Pflege tätigen Personen sehen wir ebenso die Notwendigkeit einer auf das Gesundheitswesen spezialisierten Informatik.

Die vorliegende Arbeit zeigt den Bedarf an fundierten Lehreinheiten zu digitalen und assistiven Technologien in der Ausbildung der Gesundheitsfachberufe. Die Bereitschaft der Auszubildenden dazu konnte ebenso aufgezeigt werden. Es mangelt an Wissen und Kompetenz für den effektiven und bedarfsgerechten Einsatz der Systeme in der pflegerischen Versorgung. In Anbetracht der aktuellen und sich weiter verschärfenden Versorgungssituation erscheint es unabdingbar, fundierte spezifische Bildungsangebote $\mathrm{zu}$ assistiven Techniken und zur Digitalisierung zu konzipieren und in der Ausbildungs- und Prüfungsordnung zu verankern sowie in die Pflegeausbildung zu integrieren und $\mathrm{zu}$ evaluieren. Festzuhalten ist auch, dass größere Anstrengungen im edukativen Bereich der Gesundheitsfachberufe zu unternehmen sind, um die Transformationsprozesse einer digitalen Gesundheitsversorgung nachhaltig und partizipativ gestalten zu können.

Danksagung Die Autoren bedanken sich für die in mehreren Iterationen erfolgte Konzeption und Konstruktion des Fragebogens, bei Julia Voigt, Dietrich Stoevesandt, Christine Schiller, Karsten Schwarz, Thomas Frese, Susanne Saal und Ralf Becker. Für die finale Korrektur sowie Hinweise zur Diskussion der Ergebnisse und Methode geht ein Dank an Katrin Wedler, Victoria Richey und Anne-Marie Lachmund. 
Förderung Diese Arbeit entstand im Rahmen des Forschungsprojekts FORMAT des Forschungsverbunds „Autonomie im Alter“, gefördert durch das Land Sachsen-Anhalt.

Funding Open Access funding provided by Projekt DEAL.

\section{Einhaltung ethischer Richtlinien}

Interessenkonflikt C. Buhtz, D. Paulicke, S. Hofstetter und P. Jahn geben an, dass kein Interessenkonflikt besteht.

Open Access Dieser Artikel wird unter der Creative Commons Namensnennung 4.0 International Lizenz veröffentlicht, welche die Nutzung, Vervielfältigung, Bearbeitung, Verbreitung und Wiedergabe in jeglichem Medium und Format erlaubt, sofern Sie den/die ursprünglichen Autor(en) und die Quelle ordnungsgemäß nennen, einen Link zur Creative Commons Lizenz beifügen und angeben, ob Änderungen vorgenommen wurden.

Die in diesem Artikel enthaltenen Bilder und sonstiges Drittmaterial unterliegen ebenfalls der genannten Creative Commons Lizenz, sofern sich aus der Abbildungslegende nichts anderes ergibt. Sofern das betreffende Material nicht unter der genannten Creative Commons Lizenz steht und die betreffende Handlung nicht nach gesetzlichen Vorschriften erlaubt ist, ist für die oben aufgeführten Weiterverwendungen des Materials die Einwilligung des jeweiligen Rechteinhabers einzuholen.

Weitere Details zur Lizenz entnehmen Sie bitte der Lizenzinformation auf http://creativecommons.org/licenses/by/4.0/deed.de.

\section{Literatur}

Ammenwerth E, Hackl WO (2019) Topics for continuous education in nursing Informatics: results of a survey among 280 Austrian nurses. Stud Health Technol Inform 260:162-169

Bearing Point (Hrsg) (2017) Jetzt und in die Zukunft; Smarte Gesundheit in Deutschland startet (noch) nicht durch. https://www. bearingpoint.com/files/BearingPoint_Studie_Smarte_Gesundheit_ 2017.pdf. Zugegriffen: 22. Apr. 2018

Beer J, Prakash A, Mitzner T, Rogers W (2011) Understanding robot acceptance; technical report HFA-TR-1103. https://smartech. gatech.edu/bitstream/handle/1853/39672/HFA-TR-1103-Robot Acceptance.pdf. Zugegriffen: 28. Apr. 2018

Buhtz C, Paulicke D, Hirt J, Schwarz K, Stoevesandt D, Meyer G, Jahn P (2018) Robotische Systeme zur pflegerischen Versorgung im häuslichen Umfeld: Ein Scoping Review. Z Evid Fortbild Qual Gesundhwes. https://doi.org/10.1016/j.zefq.2018.09.003

Buhtz C, Paulicke D, Schwarz K, Jahn P, Stoevesandt D, Frese T (2019) Receptiveness of GPs in the south of Saxony-Anhalt, Germany to obtaining training on technical assistance systems for caregiving: a cross-sectional study. Clin Interv Aging 14:1649-1656. https:// doi.org/10.2147/CIA.S218367

Bundesministerium für Bildung und Forschung (BMBF) (Hrsg) (2018) Forschung und Innovation für die Menschen; Die Hightech-Strategie 2025. https://www.bmbf.de/pub/Forschung_und_ Innovation_fuer_die_Menschen.pdf. Zugegriffen: 2. Dez. 2018

Davis F, Bagozzi R, Warshaw P (1989) User acceptance of computer technology: a comparison of two theoretical models. Manage Sci 35:982-1003. https://doi.org/10.1287/mnsc.35.8.982

Deisner J, Hergesell J, Maibaum A (2018) Nutzerkonfiguration und konfigurierende Nutzer in ambulanten Pflegesettings. In: Weidner R, Karafillidis A (Hrsg) Dritte Transdisziplinäre Konferenz „Technische Unterstützungssysteme, die die Menschen wirklich wollen", S 25-33

Eggert S, Sulmann D, Teubner C (2018) Einstellung der Bevölkerung zu digitaler Unterstützung in der Pflege; Quantitative Bevölke- rungsbefragung von Personen ab 18 Jahren. https://www.zqp. de/wp-content/uploads/ZQP_Analyse_PflegeDigitalisierung.pdf. Zugegriffen: 15. März 2019

Elm Ev ADG, Egger M, Pocock SJ, Gøtzsche PC, Vandenbroucke JP (2007) The strengthening the reporting of observational studies in epidemiology (STROBE) statement: guidelines for reporting observational studies. Ann Intern Med 147:573-577. https://doi. org/10.7326/0003-4819-147-8-200710160-00010

Fachinger U (2012) The demand for assisting technologies in nursing and medical care: some comments. Int J Behav Healthc Res 3:135. https://doi.org/10.1504/IJBHR.2012.046725

Generalversammlung der Vereinten Nationen (2015) A/RES/70/1* Transformation unserer Welt: die Agenda 2030 für nachhaltige Entwicklung, vom 25.09.2015. https://www.un.org/Depts/ german/gv-70/band1/ar70001.pdf. Zugegriffen: 8. Dez. 2019

Goeldner M, Herstatt C, Tietze F (2015) The emergence of care robotics-A patent and publication analysis. Technol Forecast Soc Change 92:115-131. https://doi.org/10.1016/j.techfore.2014.09. 005

Grünheid E (2015) Regionale Aspekte des demografischen Wandels. https://www.bib.bund.de/Publikation/2015/Regionale-Aspektedes-demografischen-Wandels.html. Zugegriffen: 17. Dez. 2018

Haux R (2016) Technische Systeme im Pflege- und Versorgungsmix für ältere Menschen; Expertise zum 7. Altenbericht der Bundesregierung. Deutsches Zentrum für Altersfragen. https://www. siebter-altenbericht.de/fileadmin/altenbericht/pdf/Expertise_Haux. pdf. Zugegriffen: 9. März 2017

Hergesell J (2018) Technisierte Pflege als Figuration - assistive Sicherheitstechniken und die Pflege von Menschen mit Demenz. In: Wöhlke S, Palm A (Hrsg) Mensch-Technik-Interaktion in medikalisierten Alltagen. Göttingen University Press, Göttingen, S 91-102

Hergesell J, Maibaum A (2016) Assistive Sicherheitstechniken in der geriatrischen Pflege; Konfligierende Logiken bei partizipativer Technikentwicklung. In: Weidner R (Hrsg) Zweite Transdisziplinäre Konferenz „Technische Unterstützungssysteme, die die Menschen wirklich wollen“, S 59-69

Hielscher V, Kirchen-Peters S, Sowinski C (2015) Technologisierung der Pflegearbeit?; Wissenschaftlicher Diskurs und Praxisentwicklungen in der stationären und ambulanten Langzeitpflege. Pflege Ges 2015/1:5-19

Hielscher V, Richter N (2014) Technikeinsatz und Arbeit in der Altenpflege; Ergebnisse einer internationalen Literaturrecherche. Institut für Sozialforschung und Sozialwirtschaft. https:// www.iso-institut.de/wp-content/uploads/2018/11/iso-Report_Nr. 1_Hielscher_Technikeinsatz_2014.pdf. Zugegriffen: 28.022018

Hübner U, Egbert N, Hackl W, Lysser M, Schulte G, Thye J, Ammenwerth E (2017) Welche Kernkompetenzen in Pflegeinformatik benötigen Angehörige von Pflegeberufen in den D-A-CH-Ländern?; Eine Empfehlung der GMDS, der ÖGPI und der IGPI. GMS Medizinische Informatik, Biometrie und Epidemiologie 13 https:// doi.org/10.3205/mibe000169

IBM (2017) SPSS Statistics. Version 25

Klein B, Graf B, Schlömer IF, Roßberg H, Röhricht K, Baumgarten S (2018) Robotik in der Gesundheitswirtschaft; Einsatzfelder und Potenziale. medhochzwei, Heidelberg

Kreuter V, Klingholz R (2012) Die demografische Zukunft von Europa. https://www.berlin-institut.org/fileadmin/user_upload/ Lebenswelten_in_Europa/Demografische_Zukunft_Europa_2012. pdf. Zugegriffen: 17. Dez. 2017

Krings B-J, Weinberger N (2017) Kann es technische Assistenten in der Pflege geben? Überlegungen zum Begriff der Assistenz in Pflegekontexten. In: Biniok P, Lettkemann E (Hrsg) Assistive Gesellschaft. Springer, Wiesbaden, S 183-201

Kuckartz U (2014) Mixed Methods; Methodologie, Forschungsdesigns und Analyseverfahren. Springer, Wiesbaden 
Locsin RC (2017) The co-existence of technology and caring in the theory of technological competency as caring in nursing. J Med Investig 64:160-164. https://doi.org/10.2152/jmi.64.160

Meißner A (2017) Technisierung der professionellen Pflege. Einfluss. Wirkung. Veränderung. In: Hagemann T (Hrsg) Gestaltung des Sozial- und Gesundheitswesens im Zeitalter von Digitalisierung und technischer Assistenz. Nomos, Baden-Baden, S 153-172

Mestheneos E (2011) Ageing in place in the European Union. Glob Ageing Issues Action 7:17-24

Paulicke D, Buhtz C, Meyer G, Jahn P (2019a) Beratungsansätze zu assistiven Technologien in der Pflege von Menschen mit Demenz; Fokusgruppenstudie mit pflegenden Angehörigen. Pflege. https:// doi.org/10.1024/1012-5302/a000701

Paulicke D, Buhtz C, Meyer G, Schwarz K, Stoevesandt D, Jahn P (2019b) Technikweiterbildungen für pflegende Angehörige Bildungs- und Beratungskonzepte $\mathrm{zu}$ assistiven Technologien für pflegende Angehörige von Menschen mit Demenz. Padua 14:255-264. https://doi.org/10.1024/1861-6186/a000511

Paulicke D, Schwarz K, Buhtz C, Stoevesandt D, Jahn P (2018) Multimodale und interprofessionelle Weiterbildungsangebote für Ärzte und Pflegefachkräfte zur Integration technologischer und robotischer Assistenz zur Versorgungssicherung für ältere Menschen mit Demenz in Sachsen-Anhalt. In: FHS St.Gallen (Hrsg) Forschungswelten 2018. Vielfalt leben - Offenheit erhalten, S 133-134
R Core Team (2017) R. A language and environment for Statistical Computing. Version 3.3.3. Vienna, Austria: R Foundation for Statistical Computing. https://R-projekt.org

Roland Berger GmbH (2017) ePflege - Informations- und Kommunikationstechnologie für die Pflege; Studie im Auftrag des Bundesministeriums für Gesundheit. https://www.rolandberger.com/ de/Publications/pub_epflege.html. Zugegriffen: 11. Febr. 2018

Rothgang H, Müller R (2018) BARMER Pflegereport 2018. BARMER. https://www.barmer.de/blob/170372/9186b971babc3f802 67fc329d65f8e5e/data/dl-pflegereport-komplett.pdf. Zugegriffen: 11. Dez. 2018

SoSci Survey (2018) München: SoSci Survey GmbH. https://www. soscisurvey.de/

Venkatesh V, Morris M, Davis G, Davis F (2003) User acceptance of information technology: toward a unified view. Mis Q 27:425. https://doi.org/10.2307/30036540

Zegelin A, Meyer G (2018) Roboter gegen Personalengpässe in der Pflege? Pflege 31:61-62. https://doi.org/10.1024/1012-5302/ a000607

Hinweis des Verlags Der Verlag bleibt in Hinblick auf geografische Zuordnungen und Gebietsbezeichnungen in veröffentlichten Karten und Institutsadressen neutral. 